Volume 7 • No. 14 - July 182011

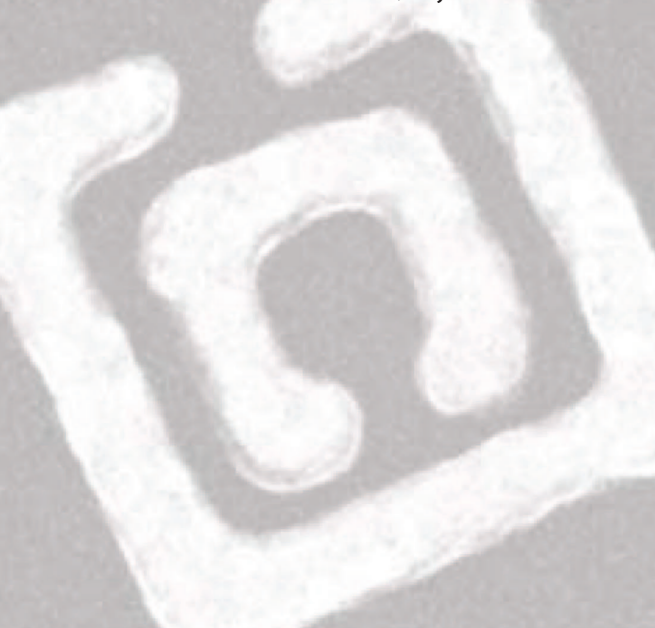

www.small-journal.com

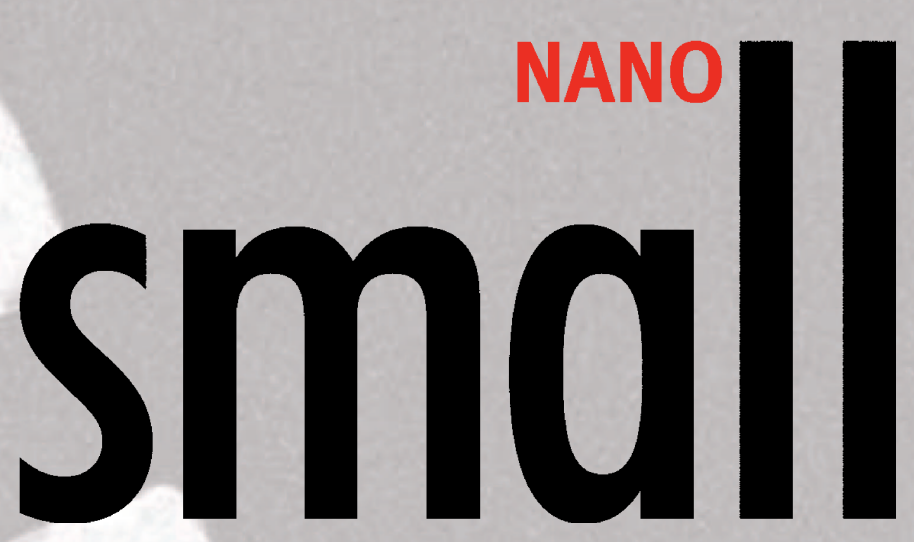

MICRO

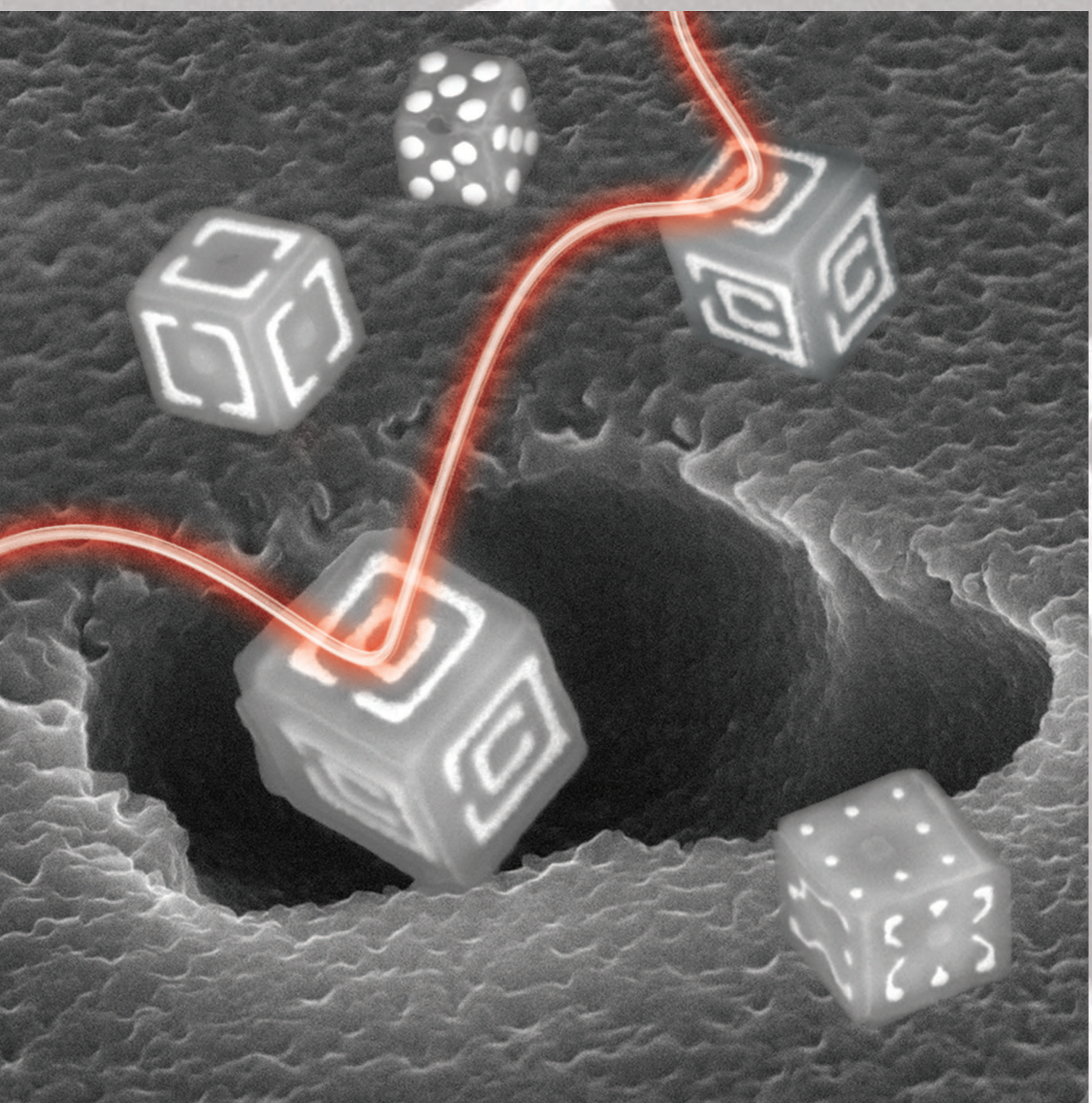

$14 / 2011$

(W)WILEY-VCH

Nanoscale Origami for 3D Optics

D. H. Gracias et al. 


\title{
Nanoscale Origami for 3D Optics
}

\author{
Jeong-Hyun Cho, Michael D. Keung, Niels Verellen, Liesbet Lagae, \\ Victor V. Moshchalkov, Pol Van Dorpe, and David H. Gracias*
}

Origami, or the ancient Japanese art of paper folding allows the transformation of flat sheets into macroscale 3D structures. ${ }^{[1]}$ Parallel wafer-scale origami-inspired approaches are especially important at the nanoscale since they can be utilized to generate precisely patterned polyhedral nanostructures that can be challenging to fabricate using conventional layer-by-layer lithographic methods. In principle, if optical or electronic elements can be patterned in two dimensions and subsequently folded up into three dimensions (Figure 1a,b), they could enable novel electronic and optical functionalities while retaining small form factors. Nanoscale devices embodied with such precise $3 \mathrm{D}$ patterning would represent a major advance as compared to existing 3D nanostructures and nanoparticles, which have only very limited surface patterns. However, while macroscale folding can be achieved by hand or by using probes and automated machines, ${ }^{[2-4]}$ manual or mechanized folding becomes increasingly difficult at the smaller micro- and nanoscales. At these smaller size scales, alternate "hands-free" mechanisms are required to generate the torques needed to lift segments of the sheet outof-plane. ${ }^{[5,6]}$

One attractive approach is to utilize differential stresses within thin films to achieve spontaneous curving or folding of micro- and nanostructures. For example, roll-up technologies, which utilize intrinsic stresses (generated during deposition) in chromium $(\mathrm{Cr})^{[7,8]}$ or silicon oxide $\left(\mathrm{SiO}_{x}\right)$ constituted bilayers, ${ }^{[9]}$ heteroepitaxially deposited $\mathrm{GaAs} / \mathrm{In}_{x} \mathrm{Ga}_{1-x} \mathrm{As}$ layers ${ }^{[10]}$ and extrinsic stresses (induced postdeposition) based on tin (Sn) grain coalescence, ${ }^{[11,12]}$ have been used to form

\author{
Dr. J. H. Cho, M. D. Keung, Prof. D. H. Gracias \\ Department of Chemical and Biomolecular Engineering \\ Johns Hopkins University \\ 3400 N Charles Street \\ Baltimore, MD 21218, USA \\ E-mail: dgracias@jhu.edu \\ N. Verellen, Prof. L. Lagae, Dr. P. Van Dorpe \\ imec, Kapeldreef 75, 3001 Leuven, Belgium \\ N. Verellen, Prof. L. Lagae, Prof. V. V. Moshchalkov \\ INPAC-Institute for Nanoscale Physics and Chemistry \\ Department of Physics and Astronomy \\ K. U. Leuven Celestijnenlaan 200 D \\ B-3001 Leuven, Belgium \\ Prof. D. H. Gracias \\ Department of Chemistry \\ Johns Hopkins University \\ 3400 N Charles Street, Baltimore, MD 21218, USA
}

DOI: $10.1002 / \mathrm{smll} .201100568$ curved structures with sub-millimeter to nanoscale radii, and are well established. In contrast to roll-up techniques, which form curved structures, origami-inspired techniques require discrete folds; hence lithographic multilayer patterning is required to define rigid panels (flat regions) and hinges (folds). For example, on the microscale we have shown that the patterning of solder or Cr-constituted bilayers as hinges have enabled the spontaneous folding of polyhedra, ${ }^{[6]}$ enzymatically triggered folding and unfolding of grippers ${ }^{[13]}$ and even geometrically programmed folding of large sheets with hundreds of bidirectional folds (self-folding materials). ${ }^{[4]}$

Here, we build on earlier demonstrations of nanoscale self-folding of polyhedra ${ }^{[15,16]}$ to create cubic structures with optically active split-ring resonator (SRRs) patterns. The optical characteristics of these structures were measured and simulated, with a focus on investigating optical functionalities enabled by 3D patterning. We utilized multilayer electron-beam (e-beam) processes to pattern planar thin films with nanoscale features so that they fold-up into cubic structures. E-beam lithography is a process where a focused beam of electrons is rastered over a resist, typically polymethyl methacrylate (PMMA). The electrons cause chain scission in the PMMA and on development, grooves are formed in regions exposed to the beam; these regions can be filled with the desired material and the PMMA can be dissolved away. Our overall process flow is depicted in Figure 1; patterns are first defined using e-beam lithography and lift-off processes (Figure 1c,h). After patterning these optically or electronically relevant features, subsequent steps of e-beam lithography are utilized, in alignment registry with the underlying patterns, to define panels (Figure 1d,i) and hinges (Figure 1e,j).

To enable origami-type folds, the structures need to be released from the substrate, and this is achieved using plasma etching of the underlying silicon substrate (Figure 1f). Plasma processing parameters that yielded good results were $25 \mathrm{~W}$ AC power applied for 40-100 s (times were based on panel geometry) with gas flow rates of 3.6 and $12 \mathrm{sccm}$ for $\mathrm{O}_{2}$ and $\mathrm{CF}_{4}$, respectively. During etching, the heat generated by the exothermic etching process, causes grain coalescence or reflow of the Sn hinges. which act as self-folds by generating a torque to rotate panels and create the $3 \mathrm{D}$ nanostructure (Figure 1g,k,l). Although a thin $\mathrm{Cr}$ adhesion layer is deposited to improve adhesion of the Au patterns to the underlying Si substrate, the $\mathrm{Cr}$ was also etched during the plasma etching process, exposing the Au patterns on the outer faces of the cubes (Figure 1f,g,k,1). Figure 1k,i show SEM images of $500 \mathrm{~nm}$ cubic structures composed of $\mathrm{Al}_{2} \mathrm{O}_{3}$ (dielectric) panels and $\mathrm{Au}$ (metal) patterns. In addition to 


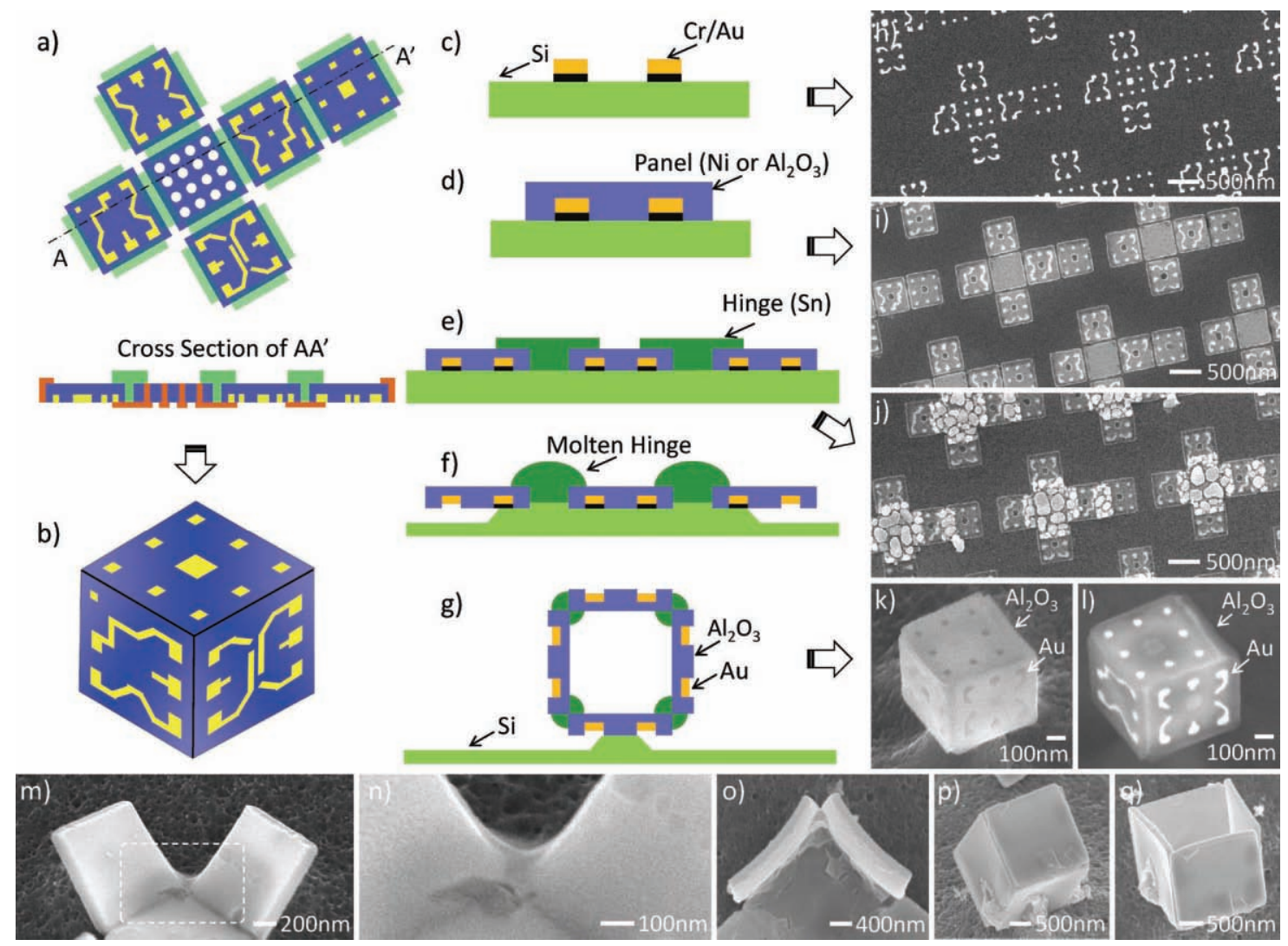

Figure 1. Conceptual schematic and experimental realization of an origami-inspired approach for fabricating precisely patterned cubes. $a, b)$ Schematic showing that self-folding can transform planar, multilayer patterned structures with optical or electronic elements into polyhedral geometries. $\mathrm{c}-\mathrm{g}$ ) Process flow and $\mathrm{h}-\mathrm{l}$ ) corresponding experimentally realized scanning electron microscopy (SEM) images showing important steps in the process; lithographic definition of $c, h$ ) the $\mathrm{Au}$ patterns, $\mathrm{d}, \mathrm{i}) \mathrm{Al}_{2} \mathrm{O}_{3}$ panels, and e,j) $\mathrm{Sn}$ hinges, in alignment registry to each other. f) Reflowing hinge during etching Si substrate and $g, k, l)$ folding up of the panels. l) This SEM image was captured using a back scatter detector which is sensitive to the atomic mass; hence the Au patterns appear brighter than the $\mathrm{Al}_{2} \mathrm{O}_{3}$ panels. $\mathrm{m}-\mathrm{q}$ ) SEM images showing that $\mathrm{Sn}$ hinges fuse at the nonfolding edges, which increases self-alignment of the edges and consequently the yield of the process.

patterning Sn at folding panel-edges, yields can be enhanced by patterning $\mathrm{Sn}$ at nonfolding panel-edges too. These hinges fuse during self-folding (Figure $1 \mathrm{~m}-\mathrm{o}$ ) and stabilize the final structure (Figure $1 \mathrm{p}-\mathrm{q}$ ). We refer to these nonfolding hinges as "locking hinges." We have observed that yields are greatly enhanced by the use of both folding and locking hinges; the self-folding hinges rotate the panels to their approximate $3 \mathrm{D}$ position and the locking hinges self-align, fuse, and seal the nonfolding edges. We also observed that the fold angle and yields were dependent on several process parameters such as the amount of Sn deposited and the plasma processing conditions.

Our choice of materials was motivated by important mechanistic parameters. Since the self-folding process is driven by heating, the hinge material needs to reflow at a relatively low temperature; we utilized $\mathrm{Sn}$ as the hinge material and either $\mathrm{Ni}$ or $\mathrm{Al}_{2} \mathrm{O}_{3}$ as panels. We observed that interatomic diffusion can cause unnecessary side reactions. For example, thin patterned copper $(\mathrm{Cu})$ films can diffuse into and react with the silicon substrate (Figure 2a), which is undesirable. We observed good $\mathrm{Sn}$ grain deposition on bare $\mathrm{Si}$ (Figure 2b); however, significant interatomic diffusion of $\mathrm{Au}$ and Sn during deposition precluded the use $\mathrm{Au}$ as panels (Figure $2 \mathrm{~b}$ ). It is important to note that intermediate wetting characteristics are also required between the hinge and panel material so that the reflowing hinge does not completely dewet or alternatively spread extensively on the panel. Dewetting was observed with reflowing silver (Ag) hinges on $\mathrm{Ni}$ (Figure 2c,d) and the structures did not fold up, while intermediate wetting was observed for reflowing Sn hinges on Ni (Figure 2e,f). Hence, on reflow, $S n$ hinges remained relatively pinned within the patterned region on the Ni panel (Figure $2 \mathrm{~g}, \mathrm{~h}$ ). In contrast, extensive spreading of $\mathrm{Sn}$ on $\mathrm{Cu}$ panels was observed causing curving of the entire structure (Figure $2 \mathrm{i}-\mathrm{j}$ ). As a consequence of our experimentation, we utilized $\mathrm{Sn} / \mathrm{Ni}$ and $\mathrm{Sn} /$ $\mathrm{Al}_{2} \mathrm{O}_{3}$; the latter allows us to define 3D nanoscale metal patterns in a dielectric medium which are important for optical applications.

The process affords some control over the fold angle, which may be required for alternate polyhedral geometries. We previously observed that the fold angle could be controlled by varying the ratio of $\mathrm{O}_{2}$ and $\mathrm{CF}_{4} \cdot{ }^{[15]} \mathrm{Here}$, another control parameter, namely manipulation of the etch time was found to result in structures with panels rotated by $45^{\circ}$ or $90^{\circ}$ angles; the extent of rotation could be correlated with the extent of Sn reflow (Figure 3). Understanding the influence 

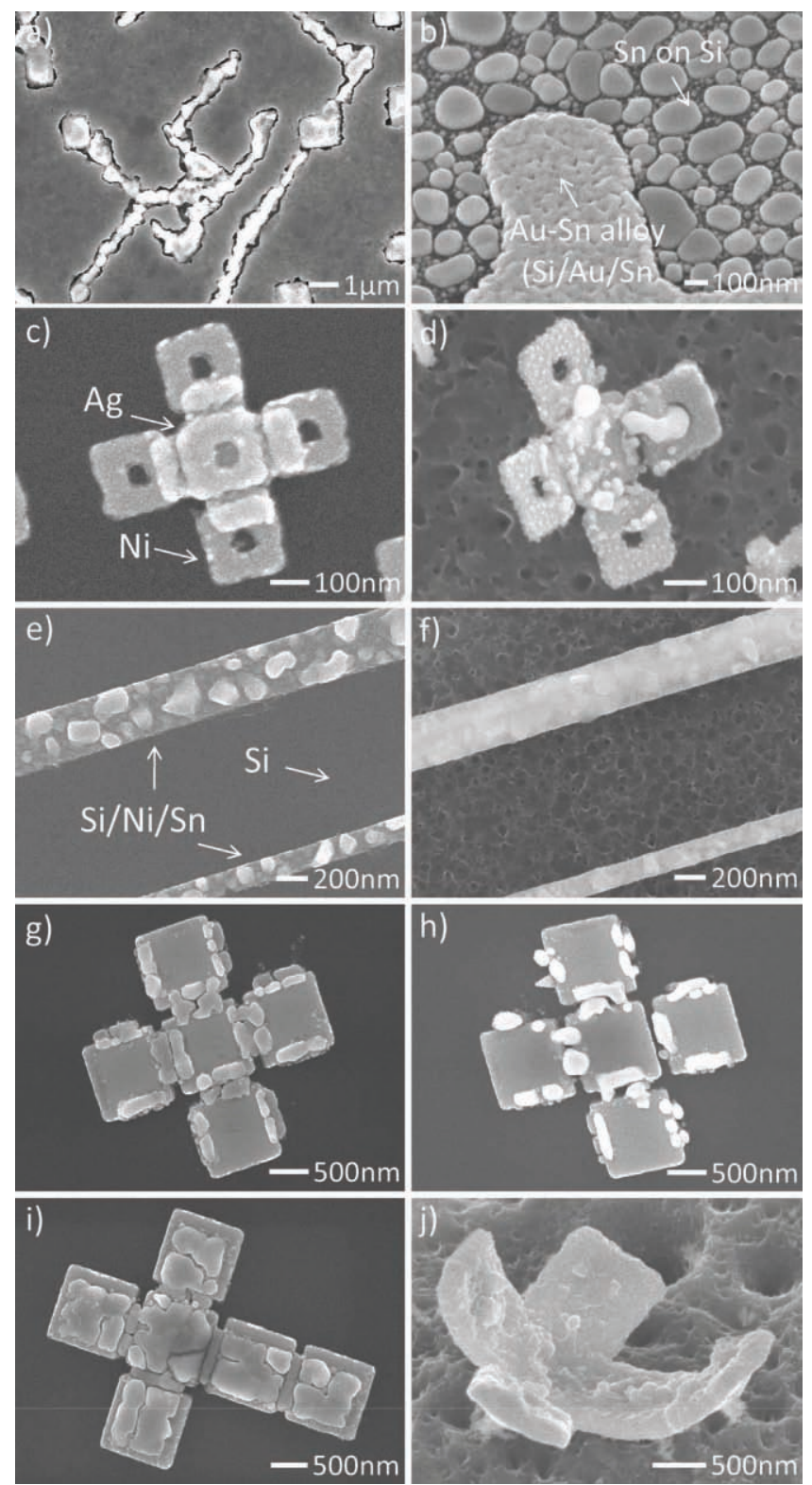

Figure 2. SEM images exploring the use of different materials for panels and hinges. a) Thin $\mathrm{Cu}$ films $(100 \mathrm{~nm})$ reacted with the Si substrate during plasma etching. b) Sn films with a thickness of $25 \mathrm{~nm}$ on bare Si and $\mathrm{Au}(16 \mathrm{~nm})$ after deposition showing interatomic diffusion for $\mathrm{Sn}$ with Au. c,d) Templates with $25 \mathrm{~nm}$ thick Ag hinges on $20 \mathrm{~nm}$ Ni panels c) as deposited, and d) after plasma etching; considerable dewetting was observed and the structures did not fold up. e,f) $\mathrm{Sn}(47 \mathrm{~nm})$ on $\mathrm{Ni}$ $(29 \mathrm{~nm})$ e) before and f) after reflow. g,h) Five-panel templates with $\mathrm{Sn}$ $(120 \mathrm{~nm})$ hinges and $\mathrm{Ni}(100 \mathrm{~nm})$ panels $\mathrm{g})$ before and $\mathrm{h})$ after reflow. Intermediate wetting of $\mathrm{Sn}$ on $\mathrm{Ni}$ allows the $\mathrm{Sn}$ to be pinned within the hinge region. We note that in order to visualize wetting, these panels were intentionally not completely released during etching (heating); hence, they did not fold-up. i,j) Six-panel templates with Sn (75 nm) hinges on $\mathrm{Cu}(100 \mathrm{~nm})$ panels; on etching, extensive spreading of $\mathrm{Sn}$ on Cu caused curvature in the structure but no discrete folds.

of various parameters on fold angle continues to be an ongoing area of research in our group.

One significant highlight of this approach is that it enables 2D patterns to be transformed into 3D patterns, as depicted in Figure 4. Here, we patterned geometric shapes, alphabets, and three types of SRRs within a single Au layer. In principle, multilayer patterns with heterogeneous materials could also be formed enabling more complex optical and electronic integration. Patterns of Au SRRs positioned along each of the $x, y$, and $z$ axes, on a dielectric $\mathrm{Al}_{2} \mathrm{O}_{3}$ cube are especially relevant for $3 \mathrm{D}$ optics. The overall sizes of the cubes are $100 \mathrm{~nm}$ (Figure 4b) and $500 \mathrm{~nm}$ (Figure 4d,f,h,j,l). We characterized optical spectra of cubes patterned with single and double SRRs (Figure 5). Figure 5a,b show simulated and experimental reflection spectra of a folded nanocube with a single SRR patterned on each of the six faces, using IR excitation. The single cube for which these spectra were collected is shown in the SEM image in the inset. The arrows indicate the corresponding polarization directions of the incident light.

The differences between simulated and experimental spectra are due to the fact that the cube was tilted slightly during etching and is not completely parallel to the substrate surface; additionally, the hinges were not included in the simulation and any metallic characteristics within the hinges are known to broaden the resonance due to strong Ohmic losses, and to result in spectral shifts. Nevertheless, the resonances are resolved experimentally and the expected polarization dependence is clearly observed. The lower-energy resonance, indicated with the black dot, is the first-order dipole mode of the SRR for polarization perpendicular to the gap region (blue curve). A charge plot of this mode is shown in the inset. The fundamental dipole resonance for polarization along the gap, the so-called magnetic mode, is located outside the experimental window, around $\lambda=5 \mu \mathrm{m}$. The pronounced resonance seen for this polarization (red curve, black square) is the second-order mode with quadrupolar charge oscillation. A corresponding study was done with double SRR patterns. Figure 5c,d show simulations and experimental realizations of spectra on the nanocube. The lower energy peak (blue curve, black dot) strongly resembles the dipole mode of the single SRR in Figure 5b (black dot), as is seen from the charge density plots. In fact, this double SRR resonance is a hybridized mode of the dipole modes in the large outer SRR and smaller inner SRR. This hybridization, first of all, leads to a red shift of the resonance. Secondly, the mode obtains a subradiant character since the dipole moments in both SRRs are oscillating out-of-phase. ${ }^{[17]}$ This leads to reduced radiative loss and linewidth collapse. The antiparallel alignment of the dipole moments can be seen in the corresponding charge density plots.

An important advantage of 3D geometry as compared to $2 \mathrm{D}$ geometry is related to the magnetic permeability $\mu$ of the structure. SRRs are known for their potential as artificial atoms in metamaterials with negative refractive index. ${ }^{[18,19]}$ The required negative $\mu$ is provided by the magnetic plasmon resonance of the SRR. However, this is only the case when the light is incident in the plane of the SRR, i.e., when the magnetic field is perpendicular to the SRR. ${ }^{[20]}$ This drastically limits the potential applications of planar 2D arrangements. A 3D cube with a proper arrangement of SRRs has the unique functionality to be the artificial atom of an isotropic (one that is functional along all spatial directions) negativeindex metamaterial. 

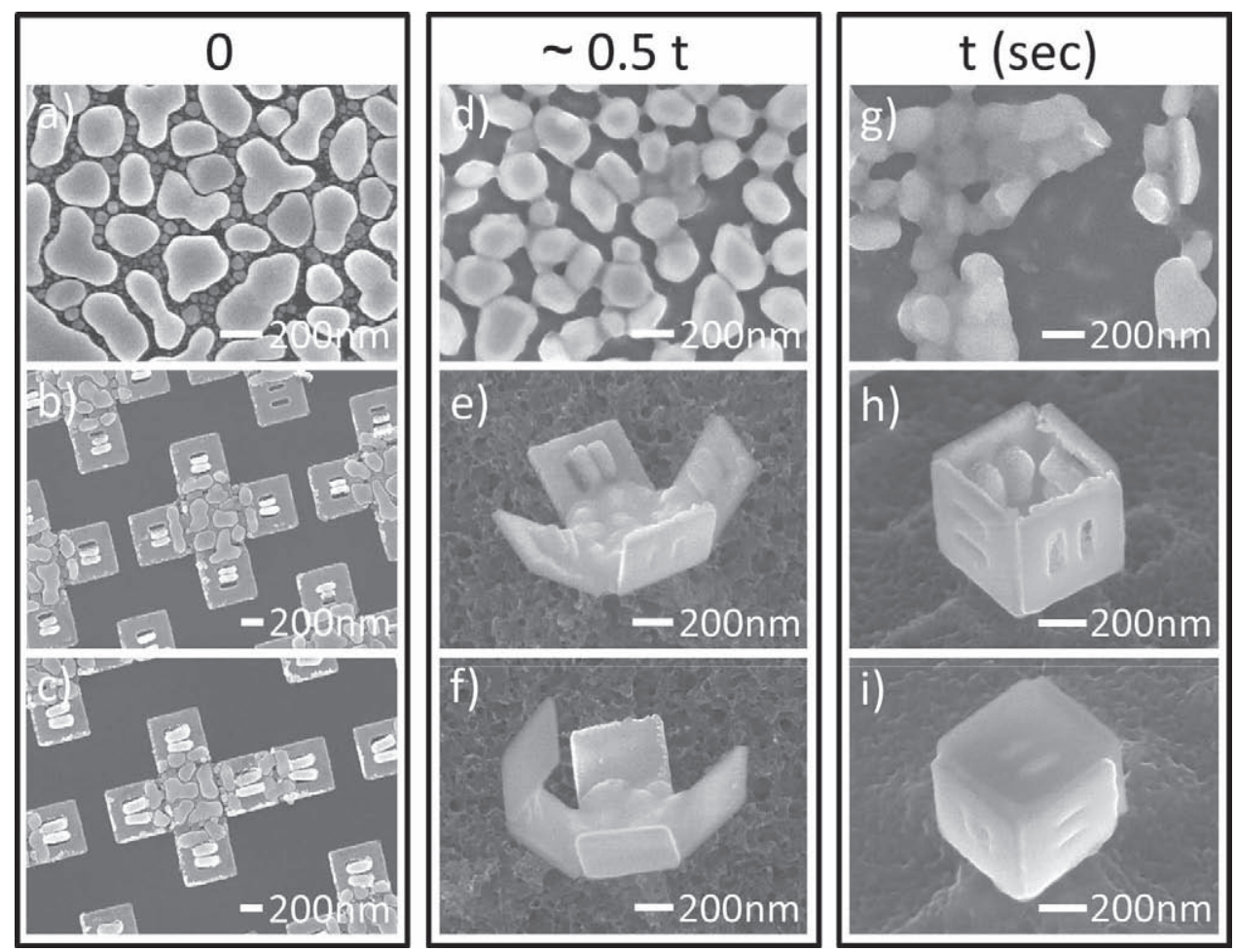

Figure 3. Control over nanoscale self-folding angle of $2 \mathrm{D}$ templates with five and six panels by varying etch times. a-c) SEM images prior to Si etching showing a) $50 \mathrm{~nm}$ thick Sn grains as deposited on a Si substrate, b) five and c) six panels with interconnected Sn hinges. $d-f$ ) At half the etch time, $\sim 0.5 t$, required to fold up the structure, d) partial grain coalescence is observed and panels in both e) five- and f) six-faced structures fold up to approximately $45^{\circ}$ angles. g-i) At the required etch times, $t, g$ ) extensive Sn grain coalescence is observed and panels in both $h$ ) five- and i) six-faced structures fold up to approximately $90^{\circ}$ angles

In addition to the SRRs explored, it should be noted that the cubes can easily be patterned with plasmonic resonators tuned to the biological tissue electromagnetic penetration window $(\lambda=700-1000 \mathrm{~nm})$, which makes them particularly interesting for applications in biosensing and in vivo surfaceenhanced Raman scattering (SERS). Cubes can for instance be designed to either sense biochemicals absorbed on its outer surface or molecules trapped on the inside of porous polyhedral structures. An example using $\mathrm{Au}$ nanocrosses ${ }^{[21]}$ is illustrated in Figure 6. A nanocross supports strong dipole and sharp quadrupole resonances. The charge distribution in the inset, indicated with the black square, reveals the quadrupolar nature of the sharp spectral feature observed at $950 \mathrm{~nm}$. In surface-plasmon-based sensors, the sensitivity is determined by two factors. The spectral shift per refractive index unit (RIU) change of the environment $(\delta \lambda / \delta n)$, and the linewidth
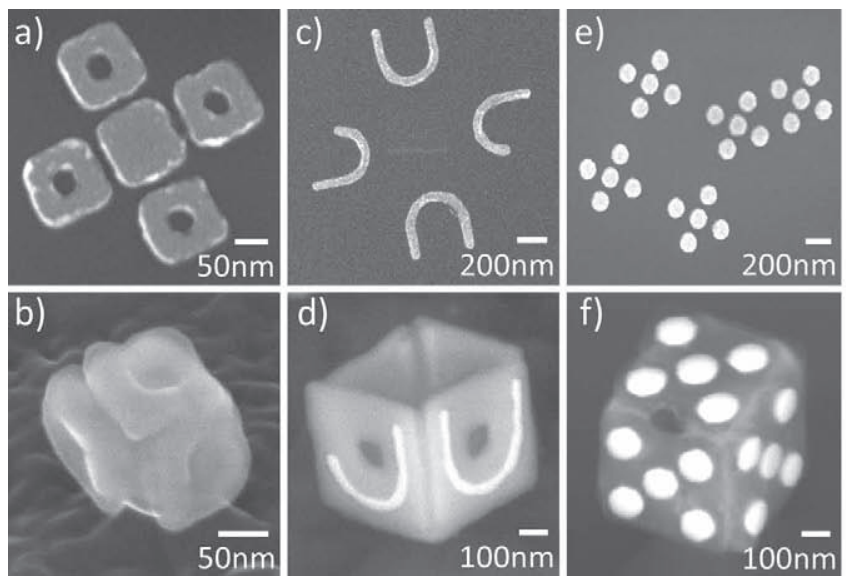
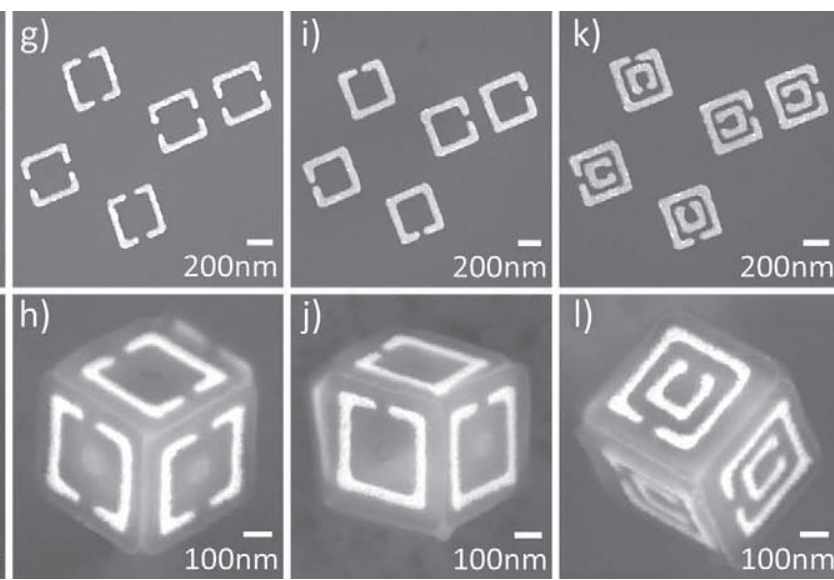

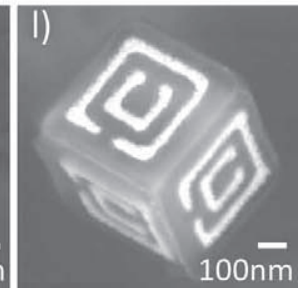

Figure 4. SEM images of the patterns and folded cubes with a-b) hollow squares, a) lithographically patterned within $13 \mathrm{~nm}$ thick Ni panels and b) the corresponding $100 \mathrm{~nm}$ folded cubic structure; c,d) $20 \mathrm{~nm}$ thick Au patterns defined with the alphabet patterns J, and U on $34 \mathrm{~nm}$ thick Ni panels; e,f) round dice-like circular $50 \mathrm{~nm}$ thick Au patterns on $50 \mathrm{~nm}$ thick $\mathrm{Al}_{2} \mathrm{O}_{3}$ panels; g,h) $50 \mathrm{~nm}$ thick Au twin loop SRRs defined on $50 \mathrm{~nm}$ thick $\mathrm{Al}_{2} \mathrm{O}_{3}$ panels; $\left.\mathrm{i}, j\right) 50 \mathrm{~nm}$ thick $\mathrm{Au}$ single loop SRRs defined on $50 \mathrm{~nm}$ thick $\mathrm{Al}_{2} \mathrm{O}_{3}$ panels; and $\left.\mathrm{k}, \mathrm{l}\right) 50 \mathrm{~nm}$ thick Au double loop SRRs defined on $50 \mathrm{~nm}$ thick $\mathrm{Al}_{2} \mathrm{O}_{3}$ panels. 

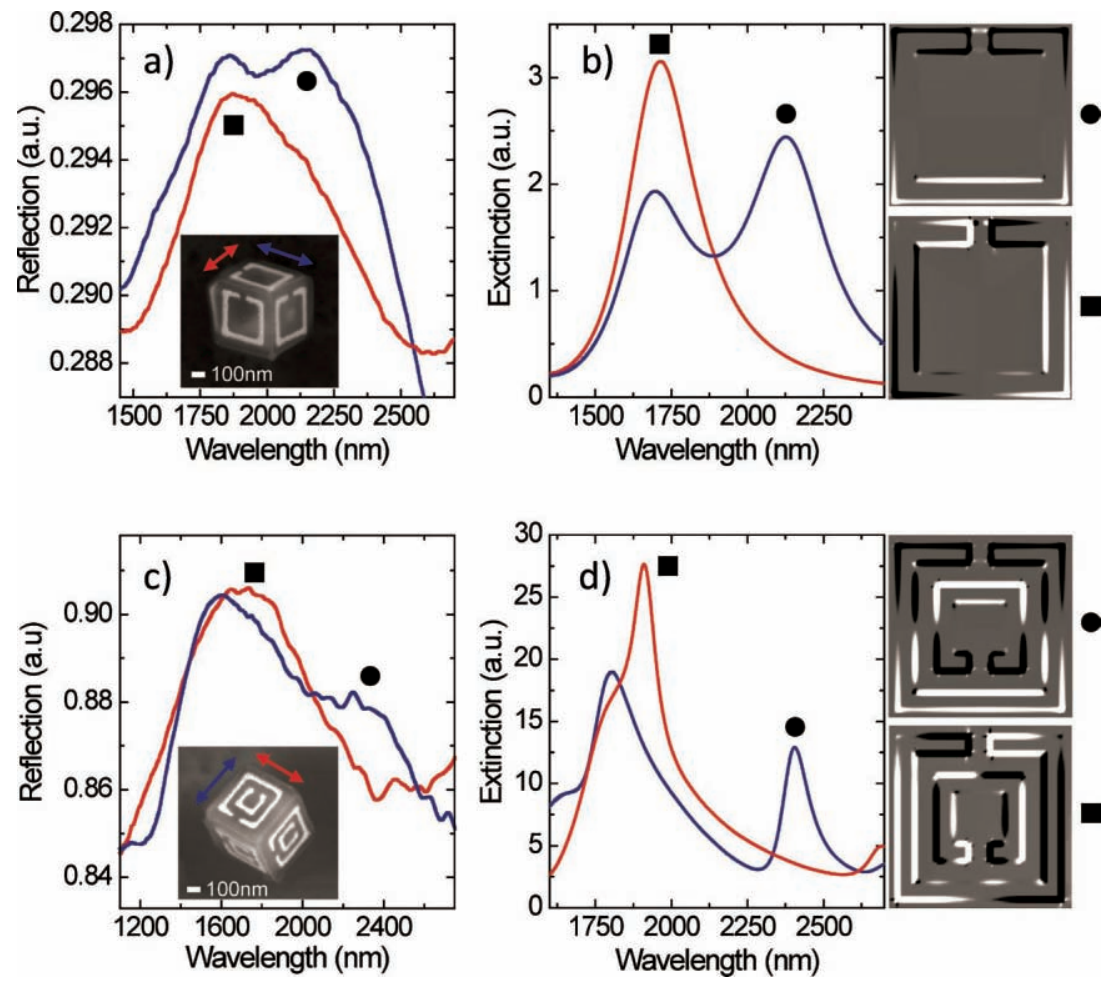

Figure 5. Experimental and simulated optical spectra of cubes with patterned $a, b)$ single and $c$,d) double SRRs on all faces. a) Single-particle Fourier-Transform (FT) IR reflection measurement of a six-faced cube decorated with single SRRs. Inset: SEM image of the measured cube. The arrows indicate the polarization of the incident light. b) Corresponding simulated extinction spectra. Insets: charge density distribution of the indicated resonances. c) Single-particle FIIR reflection measurement of a six-faced cube decorated with double SRRs. Inset: SEM image of the measured cube. The arrows indicate the polarization of the incident light. d) Corresponding simulated extinction spectra. Insets: charge density distribution of the indicated resonances.

of the detected resonance (full width at half maximum: fwhm). These two parameters are combined in one figure of merit:

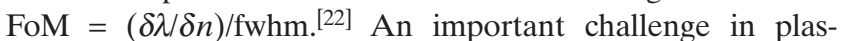
monic sensing is therefore obtaining low-loss narrow resonances. Higher-order plasmon modes, such as quadrupoles, are
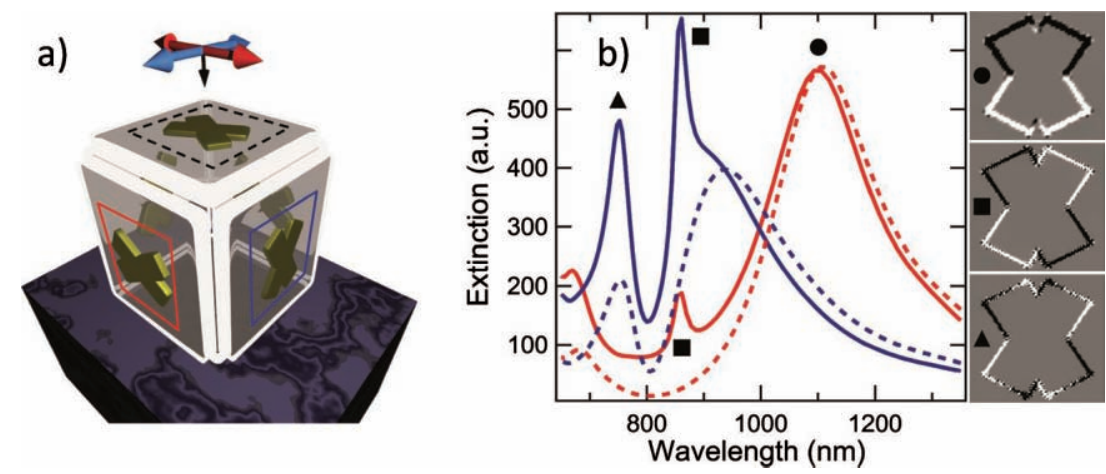

Figure 6. Direct excitation of multipolar plasmon resonances in nanocross cubes for sensing. a) Illustration of a cube decorated with crosses. b) Calculated extinction spectra for the different faces of the cube. Dashed lines obtained for the top face. These spectra correspond to a planar 2D arrangement. Full lines obtained for the side faces with the polarization direction indicated by the arrows. These spectra reveal the direct excitation of the sharp quadrupole resonance which is absent in the planar configuration. Insets: charge density distributions of the indicated resonances. (The crosses have an arm length of $200 \mathrm{~nm}$, width of $80 \mathrm{~nm}$, thickness of $50 \mathrm{~nm}$, and arm angle of $60^{\circ}$. The cube faces are $500 \mathrm{~nm} \times 500 \mathrm{~nm}$.) inherently sharp. These modes are known as dark modes since they have zero dipole moment and consequently low radiative losses. Using these dark resonances for sensing, however, requires some tricks, such as indirect excitation in the form of Fano resonances in symmetry-broken coherently coupled cavities. ${ }^{[21,23]}$ Direct excitation can only be achieved by phase retardation of the incident field along the particle volume. In planar geometries this would require an oblique incident light beam, a rather inconvenient experiment, especially when looking for single-particle detection. 3D patterning of plasmonic elements on cubes provide an excellent platform to solve these issues. As Figure 6 illustrates, crosses placed on the side faces of a cube will easily be excited at their quadrupole plasmon mode due to phase retardation of normal incident light. The arrows indicate the incident polarization, while the colored squares on the faces indicate to which cross the spectra correspond. Full lines are for side faces; dashed lines for the top face. The fact that for the top face the multipolar quadrupole mode is not excited, is one of the key advantages of a $3 \mathrm{D}$ cube over a $2 \mathrm{D}$ planar arrangement of plasmonic resonators.

In summary, nanoscale origami methods can greatly augment the capabilities of miniaturized optical devices by enabling them to be patterned in all three dimensions. We have shown considerable versatility in the types of patterns that can be formed and control over fold angle; further experimentation is required to extend this approach to other material combinations. As compared to patterning on planar surfaces, arrangements of optically active elements in polyhedral geometries can augment optical functionality. In addition to the aforementioned applications, another interesting functionality is the generation of a unique plasmonic barcode on all faces. In that way, it is possible to see the orientation of the cube by optical probing. This could be a gyroscope with plenty of opportunities for bioresearch. In our current example, we illustrate this idea by placing the cross arms at nonperpendicular angles and then rotate the crosses by $90^{\circ}$ on adjacent faces. Doing so detunes the dipole resonances, giving each adjacent face a distinguishable plasmonic response. It should also be possible to include gain materials inside the cube and likewise create a resonant cavity for nanolasing. ${ }^{[24]}$ We also envision that if large numbers of these structures can be fabricated, they can be utilized as building blocks for the aggregative self-assembly of isotropic nanostructured metamaterials. 


\section{Experimental Section}

Fabrication of Nanostructures: On a $<100>$ bare silicon wafer, $50 \mathrm{~nm}$ (for $100 \mathrm{~nm}$ cubic structure) and $100 \mathrm{~nm}$ (for 200-500 nm cubic structures) of a e-beam resist, PMMA (950 K, A2, Microchem) was spun, and the resist was baked at $185^{\circ} \mathrm{C}$ in air for $3 \mathrm{~min}$. Then, the resist was patterned using an e-beam controlled by a Raith system (Quantum Ver 4.0), and the resist was developed with a methyl isobutyl ketone (MIBK) developer diluted with isopropyl alcohol (IPA; 1:3 = MIBK:IPA) for $35 \mathrm{~s}$. On the top of the resist, an adhesion layer of $3 \mathrm{~nm} \mathrm{Cr}$ and $20-50 \mathrm{~nm}$ Au were deposited using a thermal evaporator. After deposition, the PMMA resist was dissolved in acetone for lift-off metallization. The second (for panels) and third (for hinges) step of e-beam lithography process were performed in the same manner for panels $\left(\mathrm{Ni}\right.$ or $\left.\mathrm{Al}_{2} \mathrm{O}_{3}\right)$ and hinges $(\mathrm{Sn})$. The required thickness (depending on panel size) of $\mathrm{Ni}$ and $\mathrm{Sn}$ was thermally evaporated and $\mathrm{Al}_{2} \mathrm{O}_{3}$ was deposited using an e-beam evaporator. After hinge patterning, the samples were loaded in a planar etcher (Technics PEII-A) at a base pressure of 0.15 Torr and active gases, $\mathrm{O}_{2}$ and $\mathrm{CF}_{4}$, were flowed into the etcher with the flow rate 3.6 and $12 \mathrm{sccm}$ respectively for $3 \mathrm{~min}$ in order to purge a chamber. $25 \mathrm{~W}$ plasma power (AC) was applied and sacrificial layer (Si) under the panels was etched for 40-100 s. During this time period, self-folding occurred. After that, the power was turned off, and chamber pressure was slowly increased to $1 \mathrm{~atm}$ over a period of $5 \mathrm{~min}$.

Optical Characterization: Reflection spectra were taken with a FIIR microscope (Bruker Vertex 80v + Hyperion). The incident light from a tungsten lamp is focused on the sample via a $15 \times$ magnification, NA $=0.4$ reflective Cassegrain condenser and collected in reflection with the same objective. The reflected light is further polarized, spatially filtered with a metal knife edge aperture to select only one particle, and detected with a liquid-nitrogen-cooled mercury-cadmium-telluride (MCT) detector.

Simulation Details: Calculated extinction spectra and surface charge distributions are obtained with a commercial finite-difference time-domain (FDTD) Maxwell solver ${ }^{[25]}$ using a mesh of $5 \mathrm{~nm}$. A total-field scattered-field method was used to extract the optical extinction cross sections. The charge plots are calculated at the middle cross-section of the Au structures. For the dielectric permittivity of $\mathrm{Au}$, we used a multicoefficient model fit to experimental data. ${ }^{[26]}$ We used a refractive index of $n=1.74$ for the $\mathrm{Al}_{2} \mathrm{O}_{3}$ faces. The $\mathrm{Sn}$ hinges were not incorporated in the shown simulations. Although the $\mathrm{Sn}$ hinges are retained within the experimentally realized structure, previously, we observed using energy dispersive spectroscopy (EDS) that there was an approximately $12 \%$ incorporation of fluorine within the $\mathrm{Sn}$ hinge region after reflow. However, even if we assume $\mathrm{Sn}$ to be metallic (worst case scenario), hinges were found to broaden the resonances due to high Ohmic losses in the $\mathrm{Sn}$, and to influence the spectral positions slightly.

\section{Acknowledgements}

This work was supported by the National Science Foundation (NSF) Grant No. 0854881 and 0825132 and by DuPont. N.V. acknowledges support from imec and the Methusalem funding by the Flemish Government. P.V.D. acknowledges financial support from the F.W.O. (Flanders).

This Communication is part of the Special Issue dedicated to Chad Mirkin in celebration of 20 years of influential research at Northwestern University.

[1] R. J. Lang, Origami Origami Design Secrets: Mathematical Methods for an Ancient Art, CRC Press, Natick, MA, 2003.

[2] E. E. Hui, R. T. Howe, M. S. Rodgers, in Proc. 13th Int. Conf. Microelectromech. Syst.IEEE, New Jersey 2000, 602.

[3] S. T. Brittain, O. J. A. Schueller, H. K. Wu, S. Whitesides, G. M. Whitesides, J. Phys. Chem. B 2001, 105, 347.

[4] H. J. In, S. Kumar, Y. Shao-Horn, G. Barbastathis, Appl. Phys. Lett. 2006, 88, 083104.

[5] R. R. A. Syms, E. M. Yeatman, V. M. Bright, G. M. Whitesides, J. Microelectromech. Syst. 2003, 12, 387.

[6] T. G. Leong, A. M. Zarafshar, D. H. Gracias, Small 2010, 6, 792.

[7] S. V. Golod, V. Ya. Prinz, P. Wägli, L. Zhang, O. Kirfel, E. Deckhardt, F. Glaus, C. David, D. Grützmacher, Appl. Phys. Lett. 2004, 84, 3391.

[8] P. Tyagi, N. Bassik, T. G. Leong, J. H. Cho, B. R. Benson, D. H. Gracias, IEEE/ASME J. Microelectromech. Syst. 2009, 18, 784.

[9] R. Songmuang, A. Rastelli, S. Mendach, O. G. Schmidt, Appl. Phys. Lett. 2007, 90, 091905.

[10] V. Ya. Prinz, V. A. Seleznev, A. K. Gutakovsky, A. V. Chehovskiy, V. V. Preobrazhenskii, M. A. Putyato, T. A. Gavrilova, Phys. E 2000, $6,828$.

[11] J. H. Cho, T. James, D. H. Gracias, Adv. Mater. 2010, 22, 2320.

[12] J. H. Cho, D. Datta, S. Y. Park, V. B. Shenoy, D. H. Gracias, Nano Lett. 2010, 10, 5098.

[13] N. Bassik, A. Brafman, A. M. Zarafshar, M. Jamal, D. Luvsanjav, F. M. Selaru, D. H. Gracias, J. Am. Chem. Soc. 2010, 132, 16314.

[14] N. Bassik, G. M. Stern, D. H. Gracias, Appl. Phys. Lett. 2009, 95, 091901.

[15] J. H. Cho, D. H. Gracias, Nano Lett. 2009, 9, 4049.

[16] J. H. Cho, A. Azam, D. H. Gracias, Langmuir 2010, 26, 16534.

[17] Y. Sonnefraud, N. Verellen, H. Sobhani, G. A. E. Vandenbosch, V. V. Moshchalkov, P. Van Dorpe, P. Nordlander, S. A. Maier, ACS Nano 2010, 4, 1664.

[18] V. G. Veselago, Sov. Phys. Uspekhi. 1968, 10, 509.

[19] R. A. Shelby, D. R. Smith, S. Shultz, Science 2001, 292, 77.

[20] D. R. Smith, W. J. Padilla, D. C. Vier, S. C. Nemat-Nasser, S. Schultz, Phys. Rev. Lett. 2000, 84, 4184.

[21] N. Verellen, P. Van Dorpe, C. Huang, K. Lodewijks, G. A. E. Vandenbosch, L. Lagae, V. V. Moshchalkov, Nano Lett. 2011, 11, 391 .

[22] L. J. Sherry, S.-H. Chang, G. C. Schatz, R. P. Van Duyne, B. J. Wiley, Y. Xia, Nano Lett. 2005, 5, 2034.

[23] N. Verellen, Y. Sonnefraud, H. Sobhani, F. Hao, V. V. Moshchalkov, P. Van Dorpe, P. Nordlander, S. A. Maier, Nano Lett. 2009, 9, 1663.

[24] D. J. Bergman, M. I. Stockman, Phys. Rev. Lett. 2003, 90, 027402 .

[25] Lumerical, http://www.lumerical.com, last accessed June 2011.

[26] P. B. Johnson, R. W. Christy, Phys. Rev. B 1972, 6, 4370.

Received: March 23, 2011 Published online: 